\title{
Ocupación del Oso Andino Tremarctos ornatus en el Área de Conservación Regional Choquequirao, Cusco
}

\section{Andean Bear Occupation (Tremarctos ornatus, in the Choquequirao Regional Conservation Area, Cusco}

\section{Karl H. Huaypar Loayza ${ }^{1}$, Robert Marquez ${ }^{2} \&$ Norma Jara Moscoso ${ }^{3}$}

'Gobierno Regional Cusco.ankarluz@gmail.com

${ }^{2}$ Wildlife Conservation Society Peru (WCS)

${ }^{3}$ Universidad Nacional de San Antonio de Abad del Cusco (UNSAAC)

\section{Resumen}

El conocimiento de espacios de hábitat del oso andino dentro del Área de Conservación Regional (ACR) Choquequirao, son indispensables para un manejo sostenible. El presente estudio tuvo como objetivo estudiar la ocupación de oso andino en el ACR Choquequirao, mediante modelos de ocupación para determinar el hábitat potencial de oso andino y las presiones que afectan al hábitat de la especie. Para este fin se realizaron 18 cuadrantes de $16 \mathrm{~km}^{2}$, se procesó la información SIG e imágenes satelitales, para determinar el hábitat potencial de oso andino, de acuerdo a la cobertura vegetal del ACR. Los resultados indican que el 66\% del área es su hábitat potencial. Sin embargo, esta área está siendo fragmentada por acciones antrópicas (poblaciones aledañas y carreteras). La presión que afecta en mayor medida al hábitat del oso andino dentro del ACR, es la presencia de ganado en un 40\% del ACR Choquequirao, y se superpone en un $59 \%$ al área ocupada por el oso andino, conllevando esto a un posible conflicto oso-hombre. El Área de Conservación Regional Choquequirao, cuenta con un gran porcentaje de hábitats potenciales para el oso andino Sin embargo, está siendo amenazada por factores antrópicos.

Palabras clave: ACR Choquequirao, Oso andino, cuadrantes, transectos.

\begin{abstract}
Knowledge of habitat spaces for the Andean bear within the Choquequirao Regional Conservation Area (ACR) are essential for sustainable management. The objective of this study was to study the occupation of the Andean bear in the Choquequirao ACR, using occupation models to determine the potential habitat of the Andean bear and the pressures that affect the habitat of the species. For this purpose, $1816 \mathrm{~km} 2$ quadrants were made, GIS information and satellite images were processed to determine the potential habitat of the Andean bear, according to the ACR vegetation cover. The results indicate that $66 \%$ of the area is its potential habitat. However, this area is being fragmented by anthropogenic actions (nearby towns and roads). the pressure that affects the Andean bear habitat within the ACR to a greater extent, is the presence ofcattle in $40 \%$ of the Choquequirao ACR, and it overlaps by $59 \%$ the area occupied by the Andean bear, leading to a possible conflict bear-man. The Choquequirao Regional Conservation Area-
\end{abstract}


- has a large percentage of potential habitats for the Andean bear. However, it is being threatened by anthropogenic factors. Keywords: ACR Choquequirao, Andean bear, quadrants, transects.

Keywords: ACR Choquequirao, Andean bear, quadrants, transects.

\section{Introducción}

El oso andino (Tremarctos ornatus), es la única especie de la familia Ursidae que habita en América del Sur, a lo largo de la Cordillera de los Andes, con poblaciones residentes desde Venezuela hasta Bolivia (Bernard Peyton, 1999). Su distribución en el Perú, varía entre los 250 y 4750 msnm., abarcando un área aproximada de 82,200 km² (Patton, Berlin, \& Berlin, 1982; Bernard Peyton, 1999), donde ocupa diversos ecosistemas desde los bosques secos, bosques montanos húmedos hasta súper húmedos, paramos y punas. En el Perú el oso andino muestra preferencia por el bosque montano, entre los 1900 y 2350 msnm, debido a la mayor disponibilidad de los frutos de los cuales se alimenta; así como por el bosque seco ecuatorial, en la cordillera occidental de los Andes, a partir de los $250 \mathrm{msnm}$, cuando hay disponibilidad de agua (Bernard Peyton, 1980).

El ACR Choquequirao, es la primera Área Regional del Cusco, ubicado en los distritos de Mollepata y Santa Teresa, dentro de sus objetivos tiene la conservación de los bosques (montanos húmedos, estacionalmente secos y bosques nativos) que son hábitat de importantes especies representativas tales como T. ornatus, Puma concolor, Vultur gryphus, entre otras. Y así conforman un corredor biológico entre el Santuario Histórico de Machupicchu y el santuario Nacional del Ampay (Choquequirao, 2014). El T. ornatus ha sido incluido en la Lista Roja de la Unión Mundial para la Naturaleza (UICN) en la- -lista Roja de la Unión Mundial para la Naturaleza (UICN) en la categoría de "Vulnerable" y la Convención sobre el Comercio Internacional de Especies Amenazadas de Fauna y Flora Silvestres (CITES) lo sitúa en el apéndice I, que prohíbe su comercialización (Amanzo, Chung, Zagal, \& Pacheco, 2007). A pesar de su importancia, la población del oso andino ha disminuido, debido principalmente a la pérdida de su hábitat, actualmente gran parte de su hábitat han sido reemplazado por la agricultura y ganadería bajo los 2500 m de elevación. Además, frecuentemente las áreas de páramo sobre los 3200 m están siendo remplazadas con cultivos de altura y pastoreo extensivo. Esto limita los remanentes de bosque a una banda muy estrecha y fragmentada entre los 2500 y 3000 metros.

El propósito del estudio es establecer una línea base para iniciar posteriormente un monitoreo delACR Choquequirao.

\section{Materiales y Métodos}

\section{Área de Estudio}

El estudio se desarrolló en el Área de Conservación Regional Choquequirao (103 814,39 ha), políticamente está ubicada en la jurisdicción de los distritos de Mollepata, Limatambo de la provincia de Anta y en el distrito de Santa Teresa de la provincia de La Convención en la Región Cusco. 


\section{Metodología}

Se utilizó el método de "modelos de ocupación”, para determinación de la ocupación del oso andino. Adecuado para las especies que en vida libre son difícilmente observables. Estos modelos, estiman de forma simultanea la probabilidad de ocupación $(\Psi)$ y la probabilidad de detección (p) de especies a partir de múltiples repeticiones sobre un transecto de muestreo en sitios establecidos, donde la detección se representa con "1" y la no detección con "0" (Mackenzie et al. 2006). Los datos de estas repeticiones se pueden resumir en una matriz binaria donde las columnas representan las repeticiones y las filas los sitios de búsqueda(Santiago, 2013).

Las áreas de acción promedio reportadas para el oso andino oscilan entre $14.8 \mathrm{Km}^{2}$ para hembras y $59.1 \mathrm{Km}^{2}$ para machos, y al establecer cuadrantes de 4 x $4 \mathrm{~km} 2(16 \mathrm{~km} 2)$ se mejora el muestreo (Goldstein et al., 2013). Se engrilla el área del ACR en cuadrantes de 4x4 $\mathrm{m}^{2}$ y a su vez se subdivide en 16 subcuadrantes de $1 \mathrm{x} 1 \mathrm{~km}^{2}$ para posteriormente elegir $\mathrm{y}$ evaluar solo 4 subcuadrantes. Dentro de cada subcuadrante se realiza transectos de $1,8 \mathrm{Km}$ (3 transectos de 600 metros) (Goldstein etal., 2013)(Fig. 1).

\section{Trabajo de gabinete I}

El trabajo pre-salida a campo fue elaborar el mapa de áreas potenciales de oso, dentro del ACR y determinar los cuadrantes seleccionados. En el proceso de definición de criterios para determinar las áreas potenciales de distribución del oso andino, fue necesario conocer la información de cobertura de vegetación del área, con el fin de establecer un primer criterio de acuerdo con los ecosistemas, toda esta información- -mencionada fue obtenido de fuentes secundarias; Shapes que el Gobierno Regional Cusco, tiene mediante el SINIA.

\section{- Evaluación deárea de estudio}

El ACR tiene una geografía muy heterogenia, desde los 1450 - 5750 msnm (10 zonas de vida y 9 coberturas vegetales) y la evaluación se realizó a través de un análisis de sistemas de información geográfica, de hábitat potencial de la especie basado en información histórica de hábitats en el cual la especies se ha reportado.

- Selección de diseño de muestreo: se evalúa el número de cuadrantes con más del 50\% de área con probabilidad de presencia de la especie. se establecieron 18 cuadrantes de acuerdo a la accesibilidad al lugar y el mínimo requerido para los análisis estadísticos (Goldstein etal., 2013).

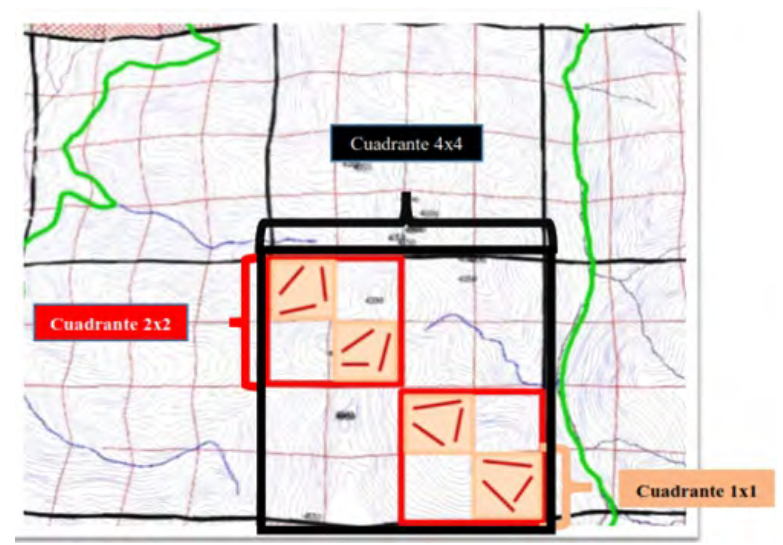

Figura 1. Determinación de cuadrantes y transectos.

\section{Trabajo de campo}

Se realizaron en total 10 salidas a campo (7 realizados por el equipo del ACR Choquequirao y 3 SERNANP Machupicchu)- 
bajo un mismo formato se colocó la información recaudada en el cuaderno de campo donde se detallaba, localidad, código, coordenadas, observaciones; en cada transecto se considera:

\section{Tipos de señales a considerar:} Comederos, marcas de ascenso, señales de rascaderos, excrementos y nidos.

Presiones: Se determinó las amenazas y ocupación de otras especies dentro de cada transecto caracterizándolos en tres tipos de impacto: ganado, tala e incendio. Toda esta información es anotada en el cuaderno de campo (Goldstein etal., 2013).

\section{Trabajo de gabinete II}

Organizar la información recolectada (Track, waypoint, fotos) en una base de datos establecida por el protocolo de la WCS (Goldstein etal., 2013), dar tratamiento estadístico con el paquete estadístico de "Presence" que calcula la ocupación del oso andino dentro del ACR.(Márquez, Navarrete, García, \& Goldstein, 2016).

- Estimación de ocupación y desviación estándar

Se realizó una matriz de datos de presencia/ausencia ( 1 si está presente y 0 si esta ausente) en cada cuadricula. Esta matriz es ingresada al programa PRESENCE, para el análisis correspondiente(presencia y/o ausencia) (Goldstein etal., 2013).

\section{Resultados}

El hábitat potencial de oso andino en Choquequirao es de 69111.64 ha de acuerdo al SIG de cobertura vegetal. Este hábitat potencial representa el $66.6 \%$ del total del área del ACR Choquequirao, comprendiendo seis tipos de coberturas vegetales distribuidos en toda el área.
El mapa muestra dos grandes parches ubicados al Este y Oeste, que ha sido separados por el nevado (abra Yanama) (Mapa 1), Así mismo, se observa que el hábitat ha sido fragmentado en 4 zonas (ubicados al N-O, S-O, $\mathrm{S}-\mathrm{E}$ y $\mathrm{N}$-E) debido al factor antrópico (Poblaciones y carreteras).
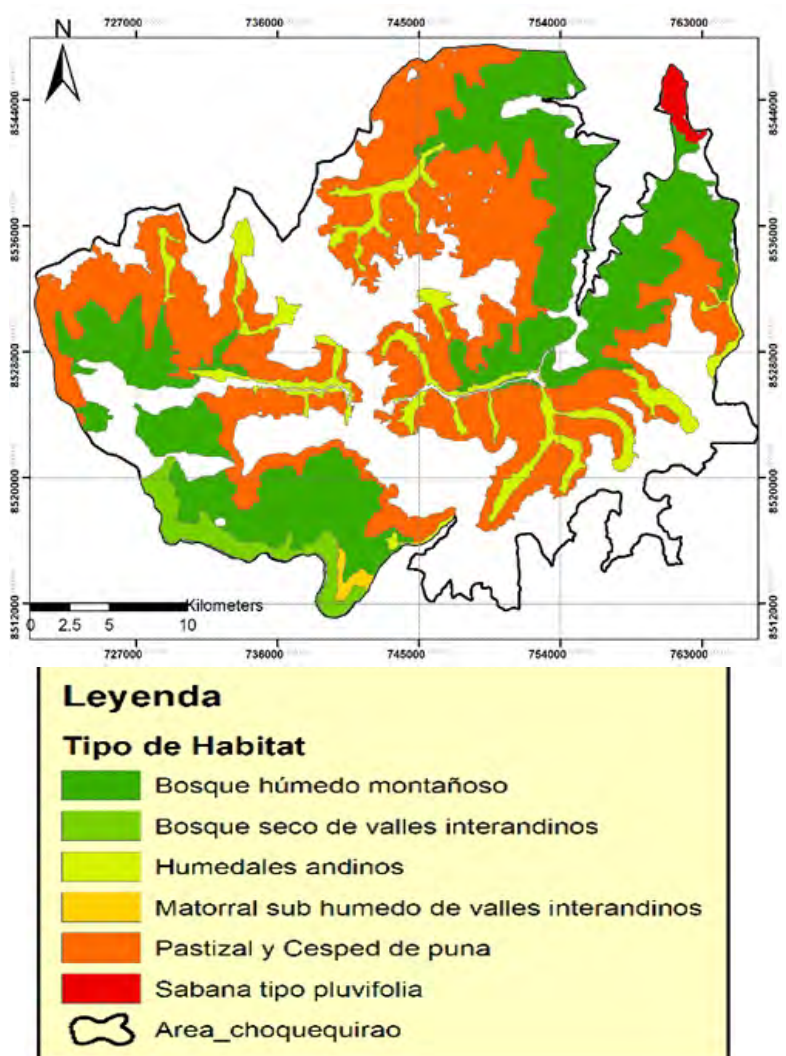

Figura 2. Hábitat Potencia de Oso Andino dentro del ACR Choquequirao.

Los ecosistemas que son parte del hábitat de la especie en estudio son Bosque húmedo montañoso, Bosque seco de valles interandinos, Matorral sub húmedo de valles interandinos, Sabana tipo pluvifolia, Pastizal y Césped de puna (Tabla 1). 


\begin{tabular}{|l|l|l|}
\hline $\mathbf{N}^{\mathbf{0}}$ & Cobertura Vegetal & \multicolumn{1}{c|}{ Área(Ha) } \\
\hline $\mathbf{1}$ & Pastizal y Césped de puna & $34,000.78$ \\
\hline $\mathbf{2}$ & Bosque húmedo montañoso & $25,500.44$ \\
\hline $\mathbf{3}$ & Humedales andinos & $6,366.36$ \\
\hline $\mathbf{4}$ & Bosque seco de valles interandinos & $2,339.45$ \\
\hline $\mathbf{5}$ & Sabana tipo pluvifolia & 583.68 \\
\hline $\mathbf{6}$ & $\begin{array}{l}\text { Matorral sub húmedo de valles } \\
\text { interandinos }\end{array}$ & 320.92 \\
\hline & $\begin{array}{l}\text { Total de Hábitat Potencial } \\
\mathbf{2}\end{array}$ & $69,111.64$ \\
\hline
\end{tabular}

Tabla 1. Clasificación de Hábitat Potencial de Oso Andino dentro del ACR Choquequirao, en Base a cobertura vegetal.

\section{Presencia de oso andino}

Como resultado final se realizó 18 cuadrantes de $4 \times 4 \mathrm{~km}^{2}$, dentro de estos cuadrantes se realizó 67 subcuadrantes de $1 \times 1 \mathrm{~km}^{2}$, siendo en total 201 transectos de 600m esto se expresa en 120600 metros dentro del ACR Choquequirao, se encontró señales de marca de garras, comederos, huellas, excremento y cuevas. La mayoría de las señales fueron encontradas en laderas y en bosques.

Señales: El 35\% de las señales corresponde a garras sobre árboles; los osos tienden a subir a estas, para su alimentación y construir sus encames. Son más notorias debido a que estas perduran a través de los años en la corteza de los árboles y es de fácil identificación por sus características únicas (Fig. 3).

El 25\% corresponde a comederos, que estos se localizaron en zonas donde había abundancia de puyales, el 20\% de excrementos, que normalmente se encontraban por las zonas de alimentación, $15 \%$ de huellas que se encontraron en caminos y cerca a fuentes de agua y finalmente un 5\% fue encontrado en nidos o encames, ubicado en arboles y/o cuevas.

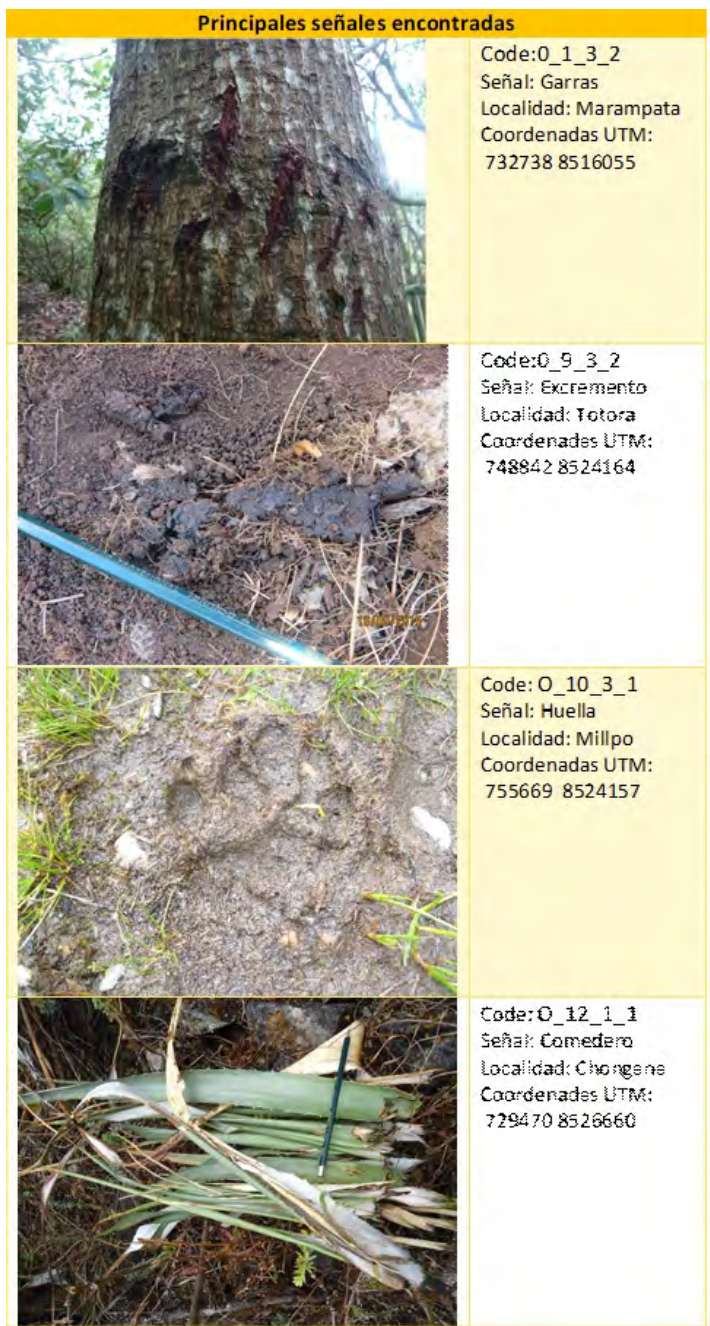

Figura 3. Registros representativos de señales encontradas en el ámbito de estudio.

La mayoría de los transectos realizados fueron ubicados en Bosque húmedo montañoso (42\%), donde las señales en su mayoría garras de ascensión, siendo este un hábitat donde se encontraron más señales de oso, seguido de Pastizal y césped de puna (33\%), humedales andinos (17\%) y finalmente Bosque seca interandino (8\%) ubicado en el sur-oeste del ACR. 


\section{Estimación de la ocupación y desviación estándar}

La ocupación del oso andino dentro de las áreas estudiadas es del 0.744, con un error estándar de 0.15 dentro de los cuadrantes de $16 \mathrm{~km}^{2}$ ( $\left.\sigma 2.746\right)($ Fig. 4).

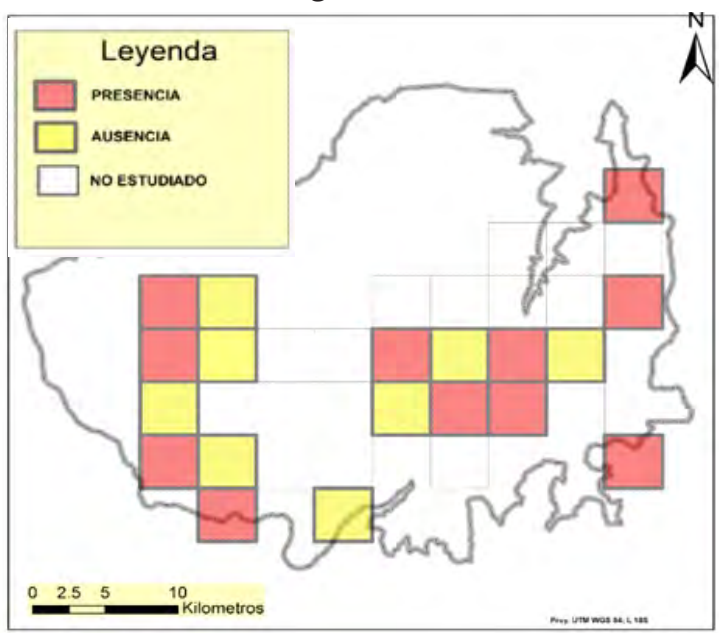

Figura 4. Mapa de ocupación de Oso Andino, del área estudiada es del 74\%.

\section{Presiones}

La presión más relevante e importante dentro del ACR es la presencia de ganado vacuno, se encontró en 44 subcuadrantes la presencia de ganado (Vacuno, equino, bovino) y dentro de estas en 92 transectos se encontró ganado. Respecto a las otras presiones (tala y quema) no son significantes.

Entonces la proporción de área ocupada por el ganado es 0.40 del total del área evaluada, siendo la principal presión que estaría afectando el hábitat de oso andino, debido a que se encontró su presencia en todas las coberturas vegetales, siendo mayoritario en césped puna, ya que los pobladores practican una agricultura extensiva.

\section{Discusiones}

El Santuario Historico de Machupicchu realizo un estudio de similar metodología y con los resultados demostrados muy distintos: teniendo en Choquequirao una ocupación de $\psi=0.74 \%$ con una detectabilidad de 0.15 mientras que en el S H. Machupicchu es $\psi=$ $0.95 \%$ con una detectabilidad igual a 0.39 (Márquez etal., 2016). Con esto se demuestra que el ACR-Choquequirao el oso andino presenta menos ocupación dentro de su área establecida, debido probablemente al estado de conservación del ACR Choquequirao, que tiene 7 años de creación, y no cuenta con infraestructura, ni con personal para la protección y conservación de sus objetos de conservación (36 años), a diferencia del S.H. Machupicchu que tiene 36 años de creación, y cuenta con todo ya antes mencionado (Puestos de control, vigilancia, guardaparques y presupuesto) además si hablamos en extensión es la tercera parte de Choquequirao.

Los indicios de presencia del oso en el ámbito de estudio están distribuidos entre 2060-4200 msnm. -(Figueroa \& Stucchi, 2013; Peyton, 1984) en estos estudios el rango de distribución oscila entre 1994 - 4170 msnm (Machupicchu), la mayoría de las señales se encuentra cerca del complejo arqueológico propiamente dicho. Los indicios dentro del ámbito de estudio, nuestras señales se encontraron entre 2060 - $4200 \mathrm{msnm}$ (Choquequirao). Si se compara con el estudios anteriores (Figueroa \& Stucchi, 2013; Peyton, 1984) en ambos el rango de distribución es entre 1994 - 4170msnm (Machupicchu), la mayoría de las señales se encuentra cerca de la ciudadela. 
Peyton (1999) y Figueroa (2016) señalan que existe un conflicto latente respecto a las actividades antrópicas (agricultura y ganadería), en la zona. El estudio identifico 3 presiones dentro del ACR Choquequirao (ganadería, quema y tala) de los cuales la más significativa es la ganadería, dentro del área. Se registró cacería de oso por estos conflictos en el sector de San Francisco - Quebrada abuela, además que Figueroa reporta una cacería en el sector de Lucma.

\section{Conclusiones}

El 66\% del ACR Choquequirao presenta un hábitat Potencial del Oso Andino Compuesto en su mayoría por el pastizal y césped de puna y bosque húmedo, mostrando de esta manera la gran variabilidad y riqueza de hábitat que presenta este ACR. La ocupación del oso andino dentro del Área de Conservación Regional Choquequirao es de un 74\%, para obtener esto se realizaron 120,600 metros en transectos dentro del ACR, encontrándose señales de marca de garras, comederos, huellas, excremento y nidos (orden decreciente), es decir se ha evidenciando que el oso andino no solo ocupa su área potencial sino que se ha visto obligado a ocupar otros sitios.

La evidencia muestra que la principal presión que sufre el hábitat del oso andino es la presencia de ganado (40\%) dentro del área de estudio, originando un conflicto oso - hombre.

\section{Agradecimientos}

Al gobierno Regional Cusco, a la Gerencia Regional de Recursos Naturales y Medio Ambiente por las facilidades de acceso al ACR. A la Asociación para la Conservación de la Cuenca Amazónica (ACCA), por el-
- financiamiento de la presente investigación. A WCS, en especial a Robert Marquez, por la capacitación en la metodología y asesoramiento en el análisis de datos. A la comunidades campesinas de Alto Salcantay por permitir el acceso las zonas y por último y no menos importante a los voluntarios y amigos de la Facultad de Ciencias Biológicas UNSAAC.

\section{Literatura citada}

Figueroa, J., \& Stucchi, M. (2013). Presencia del oso andino Tremarctos ornatus (Carnivora: Ursidae) en el Corredor de Conservación VilcabambaAmboró, sureste del Perú. Therya, 4(3), 511538. https://doi.org/10.12933/therya-13169.

Figueroa, J. (2016). Ecología y conservación del oso andino (Tremarctos ornatus) en las Áreas Naturales Protegidas del Perú.

Goldstein, I., Márquez, R., Martínez, J., Cifuentes, A., Pérez, A., Merlchor, A., ... Bianchi, G. (2013). MANUAL PARA EL MONITOREO DE OSO EN LOS PARQUES NACIONALES NATURALES DE COLOMBIA. (Alianza para la Conservacion del Oso Andino(ABCA), Ed.) (1a). Wildlife Conservation Society(WCS).

Mackenzie, D., Nichols, J., Royle, A., Pollock, K., \& James, H. (2006). Occupancy Estimation and M od e ling ( Vol.1 ). E L S E V IER. https://doi.org/10.1017/CBO9781107415324.0 04

Márquez, R., Navarrete, C., García, R., \& Goldstein, I. (2016). Evaluación de Tremarctos ornatus en el Santuario Histórico de Machupicchu , utilizando modelos de ocupación y uso (p. 172). Ecuador.

Murcia, P. (2005). Analisis de Áreas Potenciales de Distribucion de Poblaciones de Oso Andino (Tremarctos ornatus) en el Valle Del Cauca. CaliColombia. 
Peyton, B. (1984). Spectacled bear habitat use in the historical Sanctuary of Machu Picchu, Peru, 165.

Peyton, B. (1999). Spectacled bear conservation action plan. Bears: Status Survey and Conservation Action Plan, 157-198. Retrieved from http:// citeseerx.ist.psu.edu/viewdoc/download? $\mathrm{doi}=10.1 .1 .196 .5692 \& \mathrm{rep}=$ rep1\&type $=\mathrm{pdf}$

Santiago, V. (2013). Distribución potencial y conocimiento local de la nutria neotropical (Lontra longicaudiS) en la cuenca del río san juan, costa rica. 